\title{
Chitosan-Derived Three-Dimensional Porous Graphene for Advanced Supercapacitors
}

\author{
Chao Wang ${ }^{*}$ Guobin Zhong, Wei Zhao, Shijia Wu, Wei Su*, Zengfu Wei and Kaiqi Xu \\ Electric Power Research Institute of Guangdong Power Grid Cooperation, Guangzhou, China
}

Graphene for energy storage devices suffers from the preparation method and deficient quality, hindering their further widespread application. Here, we report a facile and cost-effective approach to derive three-dimensional porous graphene (3DPG) from biocompatible chitosan for massive production. Taking advantage of the large surface area, excellent electrical conductivity and high electrochemical activity of the 3DPG, an advanced symmetric supercapacitor (3DPG//3DPG SCs) is achieved by coupling two 3DPG electrodes in commercial DLC301 organic electrolyte. The device delivers a remarkable capacitance of $168.9 \mathrm{~F} \mathrm{~g}-1$ at the scan rate of $10 \mathrm{mV} \mathrm{s}-1$ and displays a

OPEN ACCESS

Edited by:

Teng Zhai,

Nanjing University of Science and

Technology, China

Reviewed by:

Xihong Lu,

Sun Yat-sen University, China

Tianyu Liu,

Virginia Tech, United States

*Correspondence:

Chao Wang

wangchaomly@163.com

Wei Su

jxhwsu@163.com

Specialty section:

This article was submitted to Electrochemical Energy Conversion and Storage,

a section of the journal

Frontiers in Energy Research

Received: 28 February 2020

Accepted: 31 March 2020

Published: 30 April 2020

Citation:

Wang C, Zhong G, Zhao W, Wu S,

Su W, Wei $Z$ and $X u K$ (2020)

Chitosan-Derived Three-Dimensional

Porous Graphene for Advanced

Supercapacitors.

Front. Energy Res. 8:61.

doi: 10.3389/fenrg.2020.00061 superior rate capability, witnessing $81.5 \%$ capacitance retention from 10 to $100 \mathrm{mV} \mathrm{s}-1$. Furthermore, the 3DPG//3DPG SCs exhibits prominent cyclic durability, as evidenced by its $96 \%$ capacitance after 10,000 cycles. This work might shed light on the probable application of graphene at industrial level for efficient energy storage.

Keywords: three-Dimensional (3D), porous, graphene, chitosan, supercapacitor

\section{INTRODUCTION}

Environmental pollution and the increasingly severe energy crisis are stimulating intensive efforts to exploit efficient energy storage devices for conversion fulfillment and storage of renewable energy sources (Wang et al., 2018; Han et al., 2019; Lu et al., 2019). In recent decades supercapacitors (SCs) have attracted particular attention, from both industry and academy, as an emerging candidate for energy storage (Hao et al., 2015; Zhang et al., 2017). This should be attributed to their advantageous features including high power density, fast charge/discharge capability, long-term cycling stability, good safety, and low maintenance cost (Yu et al., 2013; Yan et al., 2016; Chen et al., 2018). Regrettably, the dominating factor impeding the further widespread application of SCs is their low energy density, which can hardly meet the increasing energy density demands of novel electronic devices (Wang et al., 2017; Zhang Q. et al., 2018; Zhang Z. et al., 2018). The key to solving this problem lies in the rational selection of electrode materials, which determine the electrochemical performance of SCs. Among various electrode materials, carbon materials including carbon nanotubes (Sharma et al., 2015), carbon nanosphere (Cui and Zhu, 2014), active carbon (Kołodynska et al., 2016), and graphene (Wang et al., 2017; Yang et al., 2017) have been widely utilized for high-performance SCs constructions because of their impressive merits such as large surface area, prominent electrical conductivity, admirable long-term cycling stability, good mechanical properties, low production cost, and environmental friendliness (Gnanasekaran et al., 2017; Zhao et al., 2017; Guo et al., 2018). Among them, graphene has been considered as the most attractive candidate due to its high theoretical surface area $\left(2,630 \mathrm{~m}^{2} \mathrm{~g}^{-1}\right)$, impressive electrical conductivity and unique structure (Cao et al., 2011; Shen et al., 2017; Yu et al., 2017). However, the massive production of graphene with high electrochemical performance by facile and low-cost approaches is still challenging. 
Nowadays, common methods for large-scale graphene production still see visible drawbacks. For instance, the chemical vapor deposition (CVD) method allows high-quality graphene synthesis but the experimental conditions are usually harsh (Shen et al., 2017). The mechanical exfoliation method enables graphene preparation with great electrochemical properties but the product thickness of different lots is uncontrollable (Chen et al., 2012). To circumvent these issues, extensive research efforts have been devoted to the exploration of facile and reliable synthetic methods. K. Parvez et al. reported a simple electrochemical expansion approach to synthesize thin-layer graphene based on inorganic salts, and a quasi-solid-state SC with a capacitance of $18.8 \mathrm{~F} \mathrm{~g} \mathrm{~g}^{-1}$ was obtained (Shao et al., 2016). Ruoff and his co-workers designed a SC based on reduced graphene oxide by solvothermal reaction with a high capacitance of $120 \mathrm{~F} \mathrm{~g}^{-1}$ (Ruoff et al., 2012). Despite some delightful achievements, the overall performance of the cutting-edge graphene still hardly meets the demand for practical applications, especially at industry levels. Therefore, developing highly efficient approaches is very desirable for graphene preparation with excellent electrochemical properties.

Herein, we report a highly efficient approach allowing the massive production of three-dimensional porous graphene (denoted as 3DPG) as electrode materials for high-performance SCs constructions. The raw material for 3DPG derivation, the chitosan, is biocompatible, and environmentally friendly. The 3DPG electrode is facilely obtained via a three-step process, including the crosslinking of chitosan with acetic acid, the carbonization in the $\mathrm{N}_{2}$ atmosphere and the acid pickling. The porous structure and obvious graphene wrinkles of the 3DPG endow this electrode with a large surface area, which can afford a superior charge transport and adequate electrolyte diffusion into the electrode. Additionally, the nitrogen dopants in 3DPG effectively boost the reactivity and electrical conductivity by creating extrinsic defects, while the introduction of oxygen functional groups improves its electrochemical activity. As a consequence, coupling two 3DPG electrodes in commercial DLC301 organic electrolyte (denoted as 3DPG//3DPG SCs) yields an advanced symmetric SC with both a high capacitance $\left(168.9 \mathrm{~F} \mathrm{~g}^{-1}\right.$ at the scan rate of $10 \mathrm{mV} \mathrm{s}^{-1}$ ) and a superior rate capability $(81.5 \%$ capacitance retention with the scan rate increase from 10 to $100 \mathrm{mV} \mathrm{s}^{-1}$ ). Besides, the device displays extraordinary cycling durability, retaining $96 \%$ capacitance after 10,000 cycles.

\section{EXPERIMENTAL SECTION}

\section{Preparation of 3DPG Sample}

All the reagents utilized were of analytical grade and used directly without further purification. The 3DPG was synthesized by three steps, crosslinking, carbonization and pickling. Firstly, $3 \mathrm{~g}$ chitosan, $0.01 \mathrm{mmol} \mathrm{Ni}\left(\mathrm{NO}_{3}\right)_{2}$ and $1 \mathrm{mmol} \mathrm{Zn}\left(\mathrm{NO}_{3}\right)_{2}$ were dissolved in $50 \mathrm{~mL} \mathrm{H}_{2} \mathrm{O}$ under stirring at room temperature until the solution became turbid. $1.2 \mathrm{~mL}$ acetic acid was then added. After crosslinking till gel, the mixed precursor was transferred to a container $(\sim 64 \mathrm{~mL})$ and dried in a freezer dryer for $36 \mathrm{~h}$. The as-obtained precursor was soaked in a pure ethanol solution of $6 \mathrm{M} \mathrm{KOH}$ for $3 \mathrm{~h}$ and dried at $60^{\circ} \mathrm{C}$ for $3 \mathrm{~h}$. The sample was calcined under a $\mathrm{N}_{2}$ atmosphere at $500^{\circ} \mathrm{C}$ for $1 \mathrm{~h}$ and then slowly heated to $800^{\circ} \mathrm{C}$ for $1 \mathrm{~h}$. Lastly, the carbonized sample was soaked in $3 \mathrm{M} \mathrm{HCl}$ for $3 \mathrm{~h}$ at room temperature and completely rinsed with deionized water, in order to fully remove the metal ions and the residual $\mathrm{KOH}$ solution to obtain 3DPG $\left(1.21 \mathrm{mg} \mathrm{cm}^{-1}\right.$, BT25S, $0.01 \mathrm{mg}$ ).

\section{Electrode Preparation and Device Assembly}

3DPG electrodes were prepared by mixing the 3DPG powder with polyvinylidene fluoride (PVDF) binder and carbon black at a weight ratio of 8:1:1 in $\mathrm{N}, \mathrm{N}$-dimethylformamide until a homogeneous slurry was formed. Then the slurry was uniformly painted on carbon paper and dried at $80^{\circ} \mathrm{C}$ overnight. A symmetric SCs was assembled into a button battery by combining two 3DPG electrodes $(1.0 \times 1.0 \mathrm{~cm})$ and a polypropylene separator in commercial DLC301 organic electrolyte (the details can be seen in Table S1 within the Supporting Information).

\section{Material Characterizations and Electrochemical Measurements}

The morphology and microstructures of the 3DPG were analyzed by field-emission scanning electron microscopy (FESEM; JSM-6330F) and transmission electron microscope (TEM; FEI Tecnai $G^{2}$ F30). The chemical composition of the 3DPG was characterized by X-ray diffractometry (XRD; D8 ADVANCE), Raman spectroscopy (Renishaw in Via) and X-ray Photoelectron Spectroscopy (XPS, ESCALab250, Thermo VG). The Brunauer-Emmett-Teller (BET) surface area was measured with an ASAP2020M (Micromeritics Instrument Corp.). All the electrochemical studies of SCs in the potential range of $0-2.5 \mathrm{~V}$ were carried out using a button cell with a commercial DLC301 organic electrolyte. Cyclic voltammetry $(\mathrm{CV})$ and galvanostatic charge/discharge measurements (GCD) were conducted on an electrochemical workstation (CHI 760E).

\section{RESULTS AND DISCUSSION}

\section{Structural and Morphological Properties of Sample}

The 3DPG electrode is facilely obtained via a simple threestep process, including crosslinking of chitosan with acetic acid, carbonization in the $\mathrm{N}_{2}$ atmosphere, and acid pickling. Figure 1a presents an optical image of the freeze-dried chitosan after crosslinking with acetic acid, which swells into a sponge shape due to the formation of numerous porous channels during the crosslinking process. More significantly, in order to activate the graphene, the $\mathrm{Ni}\left(\mathrm{NO}_{3}\right)_{2}$ and $\mathrm{Zn}\left(\mathrm{NO}_{3}\right)_{2}$ were involved in a synthesis process and removed by sublimating at high temperature as well as soaking in $3 \mathrm{M} \mathrm{HCl}$ for $3 \mathrm{~h}$ at last, which can form a porous graphene structure with high surface area. The morphology and microstructure of the 3DPG sample was characterized by SEM and TEM. As shown in Figure 1b, the 3DPG sample exhibits notable graphene wrinkles with thickness $<1 \mathrm{~nm}$, implying the large specific surface area of the 3DPG sample. The TEM and the high-resolution transmission electron microscopy (HRTEM) images in Figures 1c,d reveal the ultrathin 

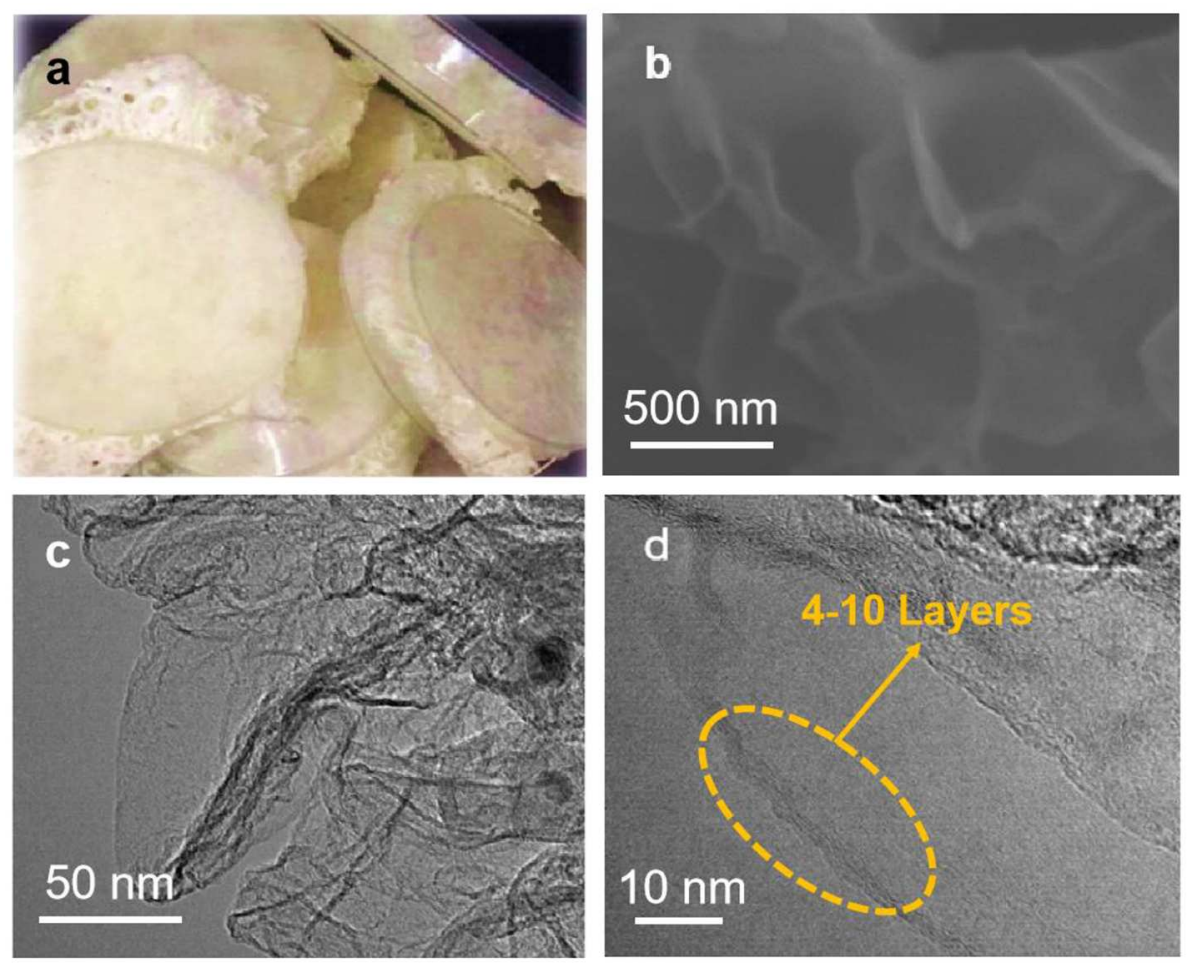

FIGURE 1 | (a) Optical image of freeze-dried chitosan after crosslinking, (b) SEM image of the 3DPG, (c) TEM image, and (d) HRTEM image of 3DPG.

wrinkles and multilayered structure of the 3DPG sample, in accordance with the SEM analysis.

The structure and crystallinity of the 3DPG sample were analyzed by conducting X-ray diffraction (XRD) measurements. As illustrated in Figure 2A, all of the diffraction peaks of the 3DPG sample can be perfectly indexed to hexagonal graphite (JCPDF \#41-1487), which is consistent with the commercial graphene (Xiao et al., 2018). No other impurity peaks were detected, indicating that the samples are pure graphene. To evaluate the specific surface area of the 3DPG sample, nitrogen adsorption-desorption measurement was carried out in Figure 2B. More encouragingly, the Brunauer-Emmett-Teller (BET) specific surface area of the 3DPG sample reaches up to $330.0 \mathrm{~m}^{2} \mathrm{~g}^{-1}$, which exceeds that of most graphene (Yang et al., 2011, 2014, 2015). Additionally, to gain insight into the chemical composition and valence state of the $3 \mathrm{DPG}$, the sample was investigated by Raman spectroscopy and X-ray photoelectron spectroscopy (XPS). The Raman spectrum in Figure 2C reveals that the two strong peaks at 1352.6 and $1585.2 \mathrm{~cm}^{-1}$ correspond to the $\mathrm{sp}^{3}$-type disordered carbon ( $\mathrm{D}$ band) and $\mathrm{sp}^{2}$-type ordered graphitic carbon ( $G$ band) (Teng et al., 2018). The large $I_{D} / I_{G}$ ratio of $\sim 0.94$ and the $2 \mathrm{D}$ band observed at $2863.5 \mathrm{~cm}^{-1}$ imply the high defect densities as well as the few-layer nature of the 3DPG sample, which are in good agreement with the SEM and HRTEM analyses (Zhou et al., 2018). The XPS spectrum of the obtained sample is revealed in Figure S1 (Supporting Information), which shows $\mathrm{C}, \mathrm{O}$, and $\mathrm{N}$ elements corresponding to atomic contents of $82.63,9.38$, and $7.99 \%$, respectively in the 3DPG sample. To further investigate the chemical state of $\mathrm{C}$ in the 3DPG sample, we analyze the $C 1$ s spectra in Figure 2D. The $\mathrm{C}$ 1s spectrum can be deconvoluted into three peaks ascribed to the $\mathrm{sp}^{2}$ carbon atoms (C-C, $284.8 \mathrm{eV}$ ), hydroxy/epoxy groups (C$\mathrm{O}, 286.0 \mathrm{eV})$, and carboxyl group $(-\mathrm{COOH}, 288.3 \mathrm{eV})$. The $\mathrm{O} 1 \mathrm{~s}$ spectrum of 3DPG in Figure 2E can be deconvoluted into two apparent peaks assigned to $\mathrm{C}-\mathrm{O}(398.6 \mathrm{eV})$ and $-\mathrm{COOH}$ groups (400.2 eV) (Zhu et al., 2015). The oxygen functional groups might endow the 3DPG with high electrochemical activity. The N 1s spectrum of 3DPG in Figure 2F displays two types of binding configurations, including pyridinic- $\mathrm{N}(511.2 \mathrm{eV})$ and graphitic$\mathrm{N}(532.9 \mathrm{eV})$. The presence of $\mathrm{N} 1 \mathrm{~s}$ and $\mathrm{O} 1 \mathrm{~s}$ spectra indicates the successful introduction of $\mathrm{N}$ and $\mathrm{O}$ in the 3DPG sample, which is capable of boosting electrical conductivity and electrochemical activity (Zhang et al., 2016; Nandan and Nanda, 2017; Xiao et al., 2018).

\section{Electrochemical Characterization of SCs}

The electrochemical performance of the symmetric SCs based on 3DPG electrodes was measured at a voltage of $2.5 \mathrm{~V}$ in a button cell with a commercial DLC301 organic electrolyte. The cyclic voltammetry $(\mathrm{CV})$ curves of 3DPG//3DPG SCs presents nearly symmetric rectangular shapes at the scan rates ranging from 10 to $100 \mathrm{mV} \mathrm{s}^{-1}$, suggesting the good capacitive performance of the device (Figure 3A). The GCD profiles of 3DPG//3DPG $\mathrm{SCs}$ at the different current densities of $0.25-3 \mathrm{~mA} \mathrm{~cm}^{-2}$ were collected in Figure 3B. It can be clearly seen that the shape of the profiles is somewhat deviated from the linear slope of an ideal supercapacitor, again demonstrating its excellent Coulombic efficiency and capacitive performance. More interestingly, the 

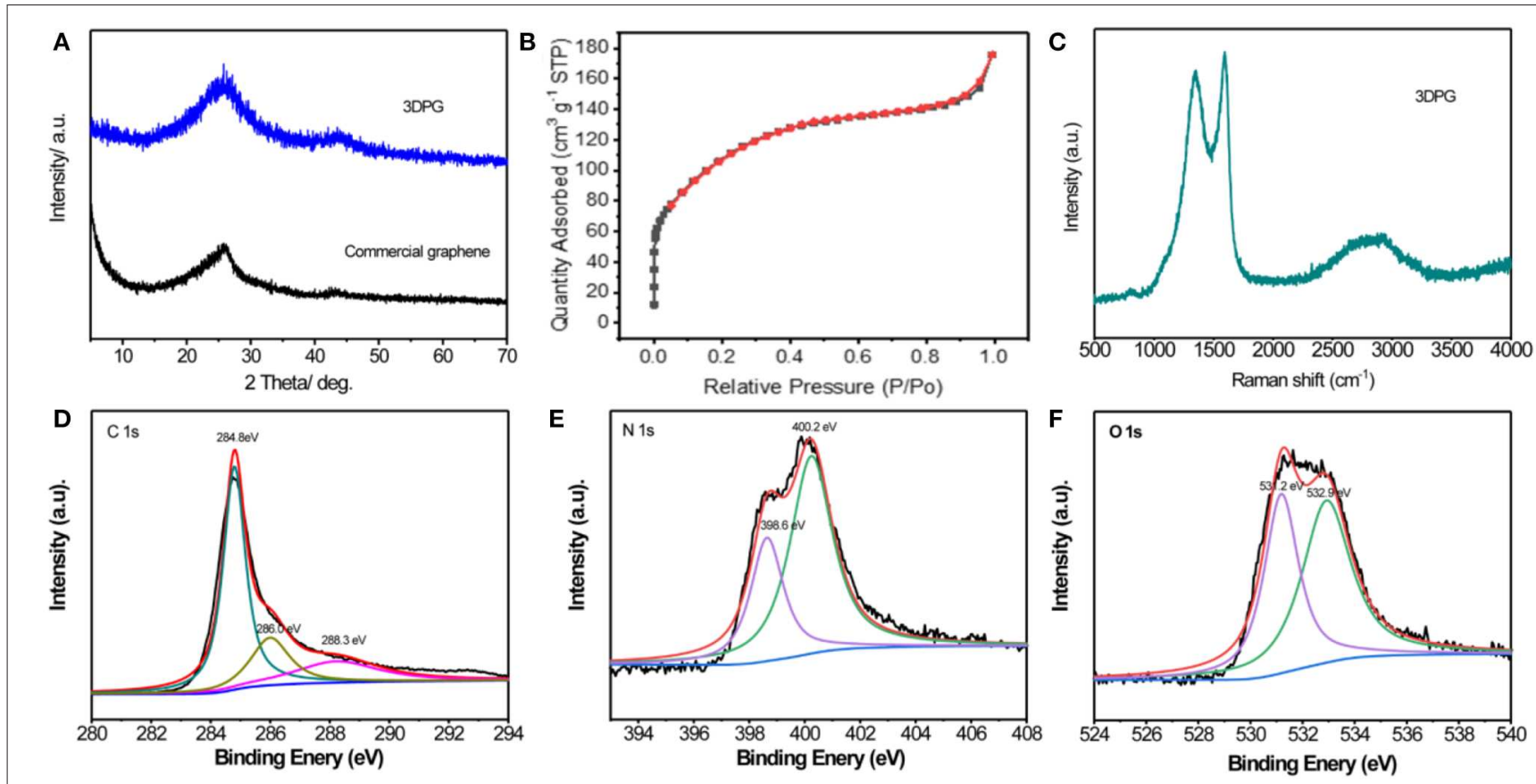

FIGURE 2 | (A) XRD patterns of 3DPG and commercial graphene, (B) Nitrogen adsorption isotherm at $77 \mathrm{~K}$, (C) Raman spectra and (D) C 1s, (E) N 1s, (F) O 1s XPS spectrums of 3DPG.
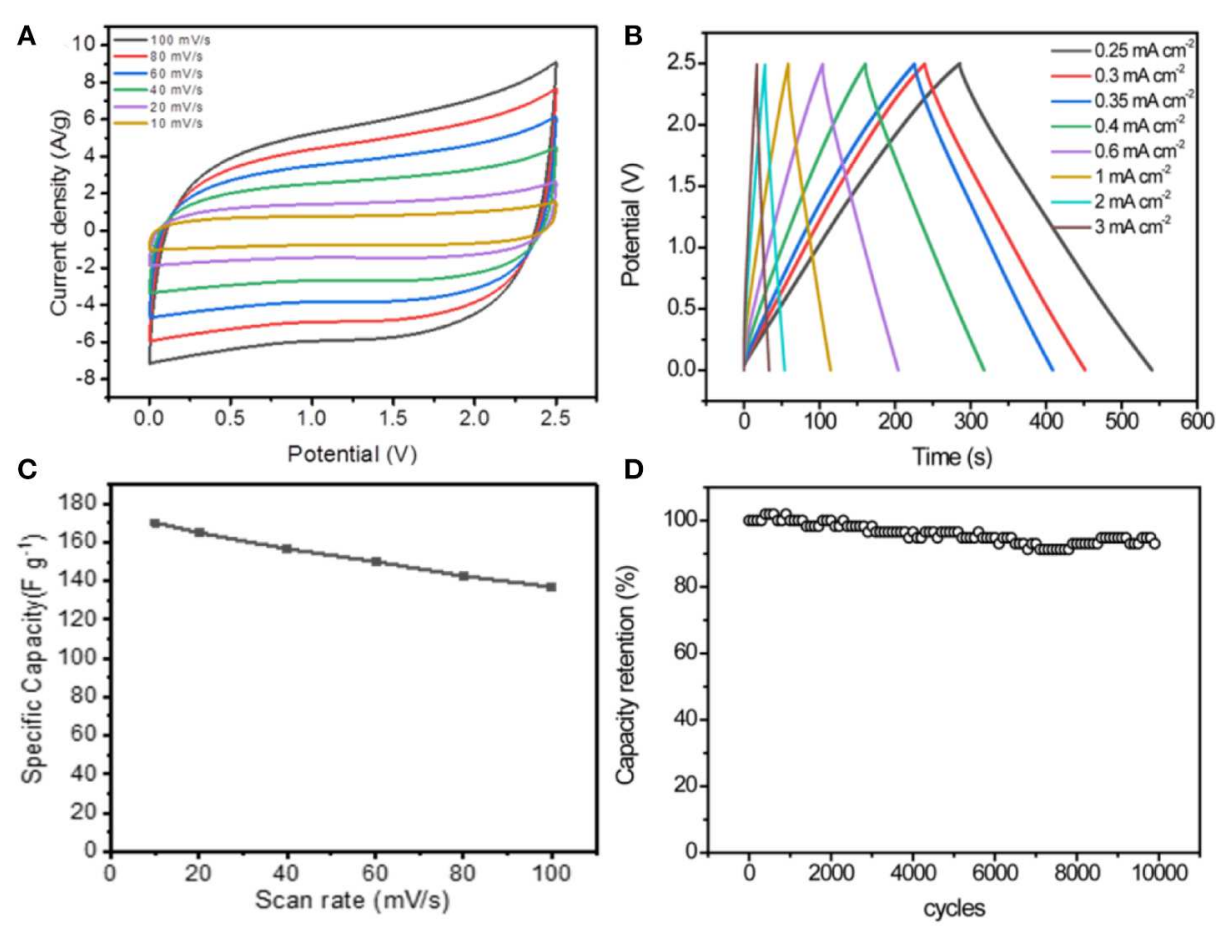

FIGURE 3 | (A) CV curves at different scan rates, (B) GCD curves at different current densities, (C) The rate performance, and (D) Cycling stability at $50 \mathrm{~mA} \mathrm{~s}^{-1}$ of 3DPG//3DPG SCs.

3DPG//3DPG SCs delivers a maximum capacitance of $168.9 \mathrm{~F}$ $\mathrm{g}^{-1}$ at the scan rate of $10 \mathrm{mV} \mathrm{s}^{-1}$ and an outstanding rate capability with $81.5 \%$ retention of its initial capacitance with the current density increase to $100 \mathrm{mV} \mathrm{s}^{-1}$ (Figure 3C). Furthermore, the cycling performance of the symmetric SC device was investigated continually by $\mathrm{CV}$ measurement at a 


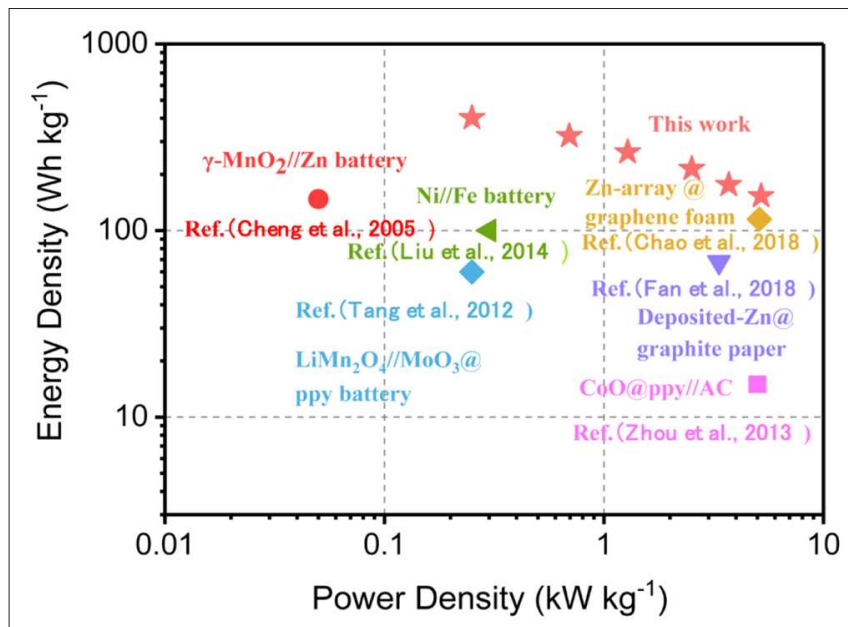

FIGURE 4 | Ragone plots of the 3DPG//3DPG SCs and other recently reported energy storage devices for comparison.

current density of $50 \mathrm{mV} \mathrm{s}^{-1}$ in Figure 3D. Significantly, this 3DPG//3DPG SC exhibits excellent cyclic durability with nearly $96 \%$ capacitance retention after 10,000 cycles. Based on the discussion above, such admirable electrochemical performance of the 3DPG//3DPG SC can be ascribed to the following reasons: (1) the $\mathrm{N}$-doping greatly improves the reactivity and electrical conductivity via the generation of extrinsic defects, endowing the electrode with a superior electrochemical performance, (2) the introduction of oxygen functional groups boosts its high electrochemical activity, and (3) Its specific porous structure and notable graphene wrinkles can provide massive electrochemically active sites (Zhu et al., 2016; Zhang et al., 2016; Antink et al., 2017; Chen et al., 2017).

The Ragone plot in Figure 4 compares the energy and power densities of the symmetric 3DPG//3DPG SCs with some other recently reported energy storage devices. Impressively, our device delivers a maximum energy density of $401.2 \mathrm{Wh}$ $\mathrm{kg}^{-1}$ at a power density of $0.25 \mathrm{~kW} \mathrm{~kg}^{-1}$. Even at a highpower density of $5.2 \mathrm{~W} \mathrm{~kg}^{-1}$, it still achieves an exceptionally high energy density of $153.2 \mathrm{Wh} \mathrm{kg}^{-1}$. Notably, the energypower characteristic of our 3DPG//3DPG SC is superior to that of recently reported energy storage devices, such as $\gamma$ $\mathrm{MnO}_{2} / / \mathrm{Zn}$ battery (156.1 Wh kg-1, $0.05 \mathrm{~kW} \mathrm{~kg}^{-1}$ ) (Cheng et al., 2005), Ni//Fe battery (100 Wh kg-1, $0.3 \mathrm{~kW} \mathrm{~kg} \mathrm{~kg}^{-1}$ ) (Liu et al., 2014), Zn-array@graphene foam (115 Wh kg-1, 5.1

\section{REFERENCES}

Antink, W., Choi, Y., Seong, K., Kim, J., and Piao, Y. (2017). Recent progress in porous graphene and reduced graphene oxide-based nanomaterials for electrochemical energy storage devices. Adv. Mater. Inter. 5:1701212. doi: 10.1002/admi.201701212

Cao, X., Shi, Y., Shi, Y., Shi, W., Lu, G., Huang, X., et al. (2011). Preparation of novel 3D graphene networks for supercapacitor applications. Small 22, 3163-3168. doi: 10.1002/smll.201100990

Chao, D., Zhu, C., Song, M., Liang, P., Zhang, X., Tiep, N., et al. (2018). A high-rate and stable quasi-solid-state zinc-ion battery with novel $2 \mathrm{D}$ layered zinc orthovanadate array. Adv. Mater. 32:1803181. doi: 10.1002/adma. 201803181
kW kg ${ }^{-1}$ ) (Chao et al., 2018), $\mathrm{LiMn}_{2} \mathrm{O}_{4} / / \mathrm{MoO}_{3} @ p p y$ battery (60 Wh kg-1, $0.25 \mathrm{~kW} \mathrm{~kg}^{-1}$ ) (Tang et al., 2012), DepositedZn@graphite paper (65.1 Wh kg-1, $3.34 \mathrm{~kW} \mathrm{~kg}^{-1}$ ) (Fan et al., 2018), CoO@ppy//AC (11.8 Wh kg ${ }^{-1}, 5.5 \mathrm{~kW} \mathrm{~kg}^{-1}$ ) (Zhou et al., 2013).

\section{CONCLUSIONS}

In conclusion, a facile and cost-effective approach was demonstrated to implement the large scale production of three-dimensional porous graphene derived from chitosan. The formation of a porous structure after the crosslinking process and the notable graphene wrinkles provide abundant electrochemically active sites for charge storage. Simultaneously, the introduction of oxygen functional groups and $\mathrm{N}$ dopants further strengthen the electrochemical activity and the electrical conductivity of the 3DPG electrode. Accordingly, an advanced symmetric SC is rationally constructed by assembling two 3DPG electrodes in commercial DLC301 organic electrolyte. The device shows an excellent capacitance of $168.9 \mathrm{~F} \mathrm{~g}^{-1}$ at the scan rate of $10 \mathrm{mV} \mathrm{s}^{-1}$ and a superior rate capability with $81.5 \%$ capacitance retention, accompanied by superior cyclic durability with $96 \%$ capacitance retention after 10,000 cycles. In consequence, such an efficient approach is of great significance in promoting the widespread application of graphene for energy storage.

\section{AUTHOR CONTRIBUTIONS}

CW and GZ conducted the experiments. WS and WZ contributed to scientific discussions and research suggestions. KX helped with writing. CW, SW, ZW, and KX performed the characterization and data analysis. All authors involved the analysis of experimental data and manuscript preparation.

\section{FUNDING}

This work was financially supported by the National Key Research and Development Program of China (Grant No. 2016YFB0901600).

\section{SUPPLEMENTARY MATERIAL}

The Supplementary Material for this article can be found online at: https://www.frontiersin.org/articles/10.3389/fenrg. 2020.00061/full\#supplementary-material

Chen, J., Duan, M., and Chen, G. (2012). Continuous mechanical exfoliation of graphene sheets via three-roll mill. J. Mater. Chem. 37:19625. doi: 10.1039/c2jm33740a

Chen, L., Lu, Y., Yu, L., and Lou, X. (2017). Designed formation of hollow particle-based nitrogen-doped carbon nanofibers for high-performance supercapacitors. Energ. Environ. Sci. 10, 1777-1783. doi: 10.1039/C7EE00488E

Chen, Y., Ni, D., Yang, X., Liu, C., Yin, J., and Cai, K. (2018). Microwave-assisted synthesis of honeycomblike hierarchical spherical Zn-doped Ni-MOF as a highperformance battery-type supercapacitor electrode material. Electrochim. Acta 278, 114-123. doi: 10.1016/j.electacta.2018.05.024

Cheng, F., Chen, J., Gou, X., and Shen, P. (2005). High-power alkaline $\mathrm{Zn}-\mathrm{MnO}_{2}$ batteries using $\gamma-\mathrm{MnO}_{2}$ nanowires/nanotubes and electrolytic zinc powder. Adv. Mater. 22, 2753-2756. doi: 10.1002/adma.200500663 
Cui, R., and Zhu, J. (2014). Fabrication of a novel electrochemical immunosensor based on the gold nanoparticles/colloidal carbon nanosphere hybrid material. Electrochim. Acta 27, 7814-7817. doi: 10.1016/j.electacta.2010.03.022

Fan, J., Xiao, Q., Fang, Y., Li, L., and Yuan, W. (2018). A rechargeable Zn/graphite dual-ion battery with an ionic liquid-based electrolyte. Ionics 25, 1303-1313. doi: 10.1007/s11581-018-2644-x

Gnanasekaran, K., Heijmans, T., Bennekom, S., Woldhuis, H., and Friedrich, H. (2017). 3D printing of CNT- and graphene-based conductive polymer nanocomposites by fused deposition modeling. Appl. Mater. Today 9, 21-28. doi: 10.1016/j.apmt.2017.04.003

Guo, Y., Xu, G., Yang, X., Ruan, K., Ma, T., Zhang, Q., et al. (2018). Significantly enhanced and precisely modeled thermal conductivity in polyimide nanocomposites with chemically modified graphene via in situ polymerization and electrospinning-hot press technology. J. Mater. Chem. C 12, 3004-3015. doi: 10.1039/C8TC00452H

Han, Y., Lu, Y., Shen, S., Zhong, Y., Liu, S., Xia, X., et al. (2019). Enhancing the capacitive storage performance of carbon fiber textile by surface and structural modulation for advanced flexible asymmetric supercapacitors. Adv. Funct. Mater. 29, 1806329-1806337. doi: 10.1002/adfm.201806329

Hao, P., Zhao, Z., Leng, Y., Tian, J., Sang, Y., Boughton, R., et al. (2015). Graphene-based nitrogen self-doped hierarchical porous carbon aerogels derived from chitosan for high performance supercapacitors. Nano Energy 15, 9-23. doi: 10.1016/j.nanoen.2015.02.035

Kołodynska, D., Krukowska, J., and Thomas, P. (2016). Comparison of sorption and desorption studies of heavy metal ions from biochar and commercial active carbon. Chem. Eng. J. 307, 353-363. doi: 10.1016/j.cej.2016.08.088

Liu, J., Chen, M., Zhang, L., Jiang, J., Yan, J., Huang, Y., et al. (2014). A flexible alkaline rechargeable $\mathrm{Ni} / \mathrm{Fe}$ battery based on graphene foam/carbon nanotubes hybrid film. Nano Lett. 14, 7180-7187. doi: 10.1021/nl503852m

Lu, Y., Zeng, S., Zhou, L., Huang, X., Zeng, Y., Zheng, D., et al. (2019). Facile synthesis of porous-carbon nanoarchitectures as advanced and durable electrodes for supercapacitors. Part. Part. Syst. Charact. 36:1900115. doi: $10.1002 /$ ppsc. 201900115

Nandan, R., and Nanda, K. (2017). Designing N-doped carbon nanotubes and $\mathrm{Fe}-\mathrm{Fe}_{3} \mathrm{C}$ nanostructures co-embedded in B-doped mesoporous carbon as an enduring cathode electrocatalyst for metal-air batteries. J. Mater. Chem. A 5, 16843-16853. doi: 10.1039/C7TA04597B

Ruoff, S., Zhang, L., Zhao, X., Stoller, D., Zhu, Y., Ji, H., et al. (2012). Highly conductive and porous activated reduced graphene oxide films for high-power supercapacitors. Nano Lett. 4, 1806-1812. doi: 10.1021/nl203903z

Shao, Y., Du, J., Li, H., Zhao, Y., and Xu, C. (2016). $\mathrm{Ni}_{0.37} \mathrm{Co}_{0.63} \mathrm{~S}_{2}$-reduced graphene oxide nanocomposites for highly efficient electrocatalytic oxygen evolution and photocatalytic pollutant degradation. J. Solid State Electr. 21, 183-192. doi: 10.1007/s10008-016-3352-y

Sharma, R., Benjwal, P., and Kar, K. (2015). Carbon Nanotubes: Synthesis, Properties and Applications. Kanpur: John Wiley and Sons, Inc. doi: 10.1002/9781119179108.ch4

Shen, C., Yi, J., Yan, X., Zhang, W., and Li, X. (2017). Effects of Cu contamination on system reliability for graphene synthesis by chemical vapor deposition method. Carbon 127, 676-680. doi: 10.1016/j.carbon.2017.11.059

Tang, W., Liu, L., Zhu, Y., Sun, H., Wu, Y., and Zhu, K. (2012). An aqueous rechargeable lithium battery of excellent rate capability based on a nanocomposite of $\mathrm{MoO}_{3}$ coated with PPy and $\mathrm{LiMn}_{2} \mathrm{O}_{4}$. Energ. Environ. Sci. 5:6909. doi: $10.1039 / \mathrm{c} 2 \mathrm{ee} 21294 \mathrm{c}$

Teng, Y., Mo, M., Yuan, L., Xue, J., and Zhao, H. (2018). Amorphous carbon-coated $\mathrm{ZnO}$ porous nanosheets: Facile fabrication and application in lithium- and sodium-ion batteries. J. All. Compd. 744, 712-720. doi: 10.1016/j.jallcom.2018.01.191

Wang, R., Lu, Y., Zhou, L., Han, Y., Ye, J., Xu, W., et al. (2018). Oxygendeficient tungsten oxide nanorods with high crystallinity: promising stable anode for asymmetric supercapacitors. Electrochim. Acta 283, 639-645. doi: 10.1016/j.electacta.2018.06.188

Wang, Z., Tammela, P., Strømme, M., and Nyholm, L. (2017). Cellulose-based supercapacitors: material and performance considerations. Adv. Energ. Mater. 18:1700130. doi: 10.1002/aenm.201700130

Xiao, X., Zeng, Y., Feng, H., Xu, K., Zhong, G., Wu, S., et al. (2018). Threedimensional nitrogen-doped graphene frameworks from electrochemical exfoliation of graphite as efficient supercapacitor electrodes. Chemnanomat. 4, 1-7. doi: 10.1002/cnma.201800452
Yan, Y., Gu, P., Zheng, S., Zheng, M., Pang, H., and Xue, H. (2016). Facile synthesis of an accordion-like Ni-MOF superstructure for high-performance flexible supercapacitors. J. Mater. Chem. A 4:19078. doi: 10.1039/C6TA08331E

Yang, N., Zhai, J., Wang, D., Chen, Y., and Jiang, L. (2017). Two-dimensional graphene bridges enhanced photoinduced charge transport in dye-sensitized solar cells. ACS Nano 2, 887-894. doi: 10.1021/nn901660v

Yang, S., Feng, X., and Mullen, K. (2011). Sandwich-like, graphene-based titania nanosheets with high surface area for fast lithium storage. Adv Mater 23, 3575-3579. doi: 10.1002/adma.201101599

Yang, Z., Jin, L., Lu, G., Xiao, Q., Zhang, Y., Jing, L., et al. (2014). Sponge-templated preparation of high surface area graphene with ultrahigh capacitive deionization performance. Adv. Funct. Mater. 24, 3917-3925. doi: 10.1002/adfm.201304091

Yang, Z., Jin, L., Lu, G., Xiao, Q., Zhang, Y., Jing, L., et al. (2015). Response to comment onsponge-templated preparation of high surface area graphene with ultrahigh capacitive deionization performance. Adv. Funct. Mater. 25, 182-183. doi: 10.1002/adfm.201403534

Yu, G., Xing, X., Pan, L., Bao, Z., and Yi, C. (2013). Hybrid nanostructured materials for high-performance electrochemical capacitors. Nano Energy 2, 213-234. doi: 10.1016/j.nanoen.2012.10.006

Yu, X., Zhang, W., Zhang, P., and Su, Z. (2017). Fabrication technologies and sensing applications of graphene-based composite films: advances and challenges. Biosens. Bioelectron. 89, 72-84. doi: 10.1016/j.bios.2016.01.081

Zhang Q, Han K, Li S, Li M, Li J, Ren K. Synthesis of garlic skin-derived 3D hierarchical porous carbon for high-performance supercapacitors. Nanoscale (2018) 10, 2427-2437. doi: 10.1039/C7NR07158B

Zhang, F., Liu, T., Li, M., Yu, M., Luo, Y., Tong, Y., et al. (2017). Multiscale pore network boosts capacitance of carbon electrodes for ultrafast charging. Nano Lett. 17, 3097-3104. doi: 10.1021/acs.nanolett.7b00533

Zhang, H., Qiu, W., Zhang, Y., and Han, Y. (2016). Surface engineering of carbon fiber paper for efficient capacitive energy storage. J. Mater. Chem. 4:18639. doi: 10.1039/C6TA08138J

Zhang, Z., Zhang, X., Feng, Y., and Sun, Q. (2018). Fabrication of porous $\mathrm{ZnCo}_{2} \mathrm{O}_{4}$ nanoribbon arrays on nickel foam for high-performance supercapacitors and lithium-ion batteries. Electrochim. Acta 260, 823-829. doi: 10.1016/j.electacta.2017.12.047

Zhao, D., Zhang, J., Fu, C., Huang, J., Xiao, D., Yuen, M., et al. (2017). Enhanced cycling stability of ring-shaped phosphorus inside multi-walled carbon nanotubes as anodes for lithium-ion batteries. J. Mater. Chem. A 6, 2540-2548. doi: 10.1039/C7TA07683E

Zhou, C., Zhang, Y., Li, Y., and Liu, J. (2013). Construction of high-capacitance 3D CoO@Polypyrrole nanowire array electrode for aqueous asymmetric supercapacitor. Nano Lett. 13, 2078-2085. doi: 10.1021/nl400378j

Zhou, F., Huang, H., Xiao, C., Zheng, S., Shi, X., Qin, J., et al. (2018). Electrochemically scalable production of fluorine-modified graphene for flexible and high-energy ionogel-based microsupercapacitors. J. Am. Chem. Soc. 140, 8198-8205. doi: 10.1021/jacs.8b03235

Zhu, G., Xi, C., Liu, Y., Zhua, J., and Shen, X. (2015). CN foam loaded with fewlayer graphene nanosheets for high-performance supercapacitor electrodes. $J$. Mater. Chem. A. 3, 7591-7599. doi: 10.1039/C5TA00837A

Zhu, J., Childress, A., Karakaya, M., Dandeliya, S., and Podila, R. (2016). Defect-engineered graphene for high-energy and high-power density supercapacitor devices. Adv. Mater. 33, 7185-7192. doi: 10.1002/adma. 201602028

Conflict of Interest: All the authors are now employed by the company Electric Power Research Institute of Guangdong Power Grid Co., Ltd. The authors declare that this study received funding from the National Key Research and Development Program of China (Grant No. 2016YFB0901600). The funder was not involved in the study design, collection, analysis, interpretation of data, the writing of this article or the decision to submit it for publication.

Copyright (c) 2020 Wang, Zhong, Zhao, Wu, Su, Wei and Xu. This is an open-access article distributed under the terms of the Creative Commons Attribution License (CC $B Y)$. The use, distribution or reproduction in other forums is permitted, provided the original author(s) and the copyright owner(s) are credited and that the original publication in this journal is cited, in accordance with accepted academic practice. No use, distribution or reproduction is permitted which does not comply with these terms. 